\title{
Bounds for the Z-spectral radius of nonnegative tensors
}

\author{
Jun $\mathrm{He}^{*}$, Yan-Min Liu ${ }^{\dagger}$, Hua Ke ${ }^{\dagger}$, Jun-Kang Tian ${ }^{\dagger}$ and Xiang $\mathrm{Li}^{\dagger}$
}

*Correspondence: hejunfan1@163.com

†Jun He, Yan-Min Liu, Hua Ke,

Jun-Kang Tian and Xiang Li

contributed equally to this

work

School of Mathematics,

Zunyi Normal College,

Zunyi 563002, Guizhou,

People's Republic of China

\section{Abstract}

In this paper, we have proposed some new upper bounds for the largest Z-eigenvalue of an irreducible weakly symmetric and nonnegative tensor, which improve the known upper bounds obtained in Chang et al. (Linear Algebra Appl 438:4166-4182, 2013), Song and Qi (SIAM J Matrix Anal Appl 34:1581-1595, 2013), He and Huang (Appl Math Lett 38:1 10-114, 2014), Li et al. (J Comput Anal Appl 483:182-199, 2015), He (J Comput Anal Appl 20:1290-1301, 2016).

Keywords: Bound, Nonnegative tensor, Z-eigenvalue

Mathematics Subject Classification: 15A18, 15A69, 65F15, 65F10

\section{Background}

Let $\mathbb{R}$ be the real field. An $m$ th order $n$ dimensional square tensor $\mathcal{A}$ consists of $n^{m}$ entries in $\mathbb{R}$, which is defined as follows:

$$
\mathcal{A}=\left(a_{i_{1} i_{2} \ldots i_{m}}\right), \quad a_{i_{1} i_{2} \ldots i_{m}} \in \mathbb{R}, \quad 1 \leq i_{1}, i_{2}, \ldots i_{m} \leq n .
$$

$\mathcal{A}$ is called nonnegative if $a_{i_{1} i_{2} \ldots i_{m}} \geq 0$. To an n-vector $x$, real or complex, we define the n-vector:

$$
\mathcal{A} x^{m-1}=\left(\sum_{i_{2}, \ldots, i_{m}=1}^{n} a_{i i_{2} \ldots i_{m}} x_{i_{2}} \ldots x_{i_{m}}\right)_{1 \leq i \leq n}
$$

and

$$
x^{[m-1]}=\left(x_{i}^{m-1}\right)_{1 \leq i \leq n} .
$$

If $\mathcal{A} x^{m-1}=\lambda x^{[m-1]}, x$ and $\lambda$ are all real, then $\lambda$ is called an H-eigenvalue of $\mathcal{A}$ and $x$ an H-eigenvector of $\mathcal{A}$ associated with $\lambda$. If $\mathcal{A} x^{m-1}=\lambda x$ with $x^{T} x=1, x$ and $\lambda$ are all real, then $\lambda$ is called a Z-eigenvalue of $\mathcal{A}$ and $x$ a Z-eigenvector of $\mathcal{A}$ associated with $\lambda$ Qi (2005), Lim (2005). See more about the eigenvalue problems of tensors in Chang et al. (2009, 2010), Qi (2007), Yang and Yang (2010, 2011), Ng et al. (2009), Zhou et al. (2013), Li et al. (2014, 2015), Hu and Huang (2012), Hu et al. (2013).

The following definition for irreducibility has been introduced in Chang et al. (2008) and $\operatorname{Lim}(2005)$.

(c) 2016 The Author(s). This article is distributed under the terms of the Creative Commons Attribution 4.0 International License (http://creativecommons.org/licenses/by/4.0/), which permits unrestricted use, distribution, and reproduction in any medium, provided you give appropriate credit to the original author(s) and the source, provide a link to the Creative Commons license, and indicate if changes were made. 
Definition 1 The square tensor $\mathcal{A}$ is called reducible if there exists a nonempty proper index subset $\mathbb{J} \subset\{1,2, \ldots, n\}$ such that $a_{i_{1}, i_{2}, \ldots, i_{m}}=0, \forall i_{1} \in \mathbb{J}, \forall i_{2}, \ldots, i_{m} \notin \mathbb{J}$. If $\mathcal{A}$ is not reducible, then we call $\mathcal{A}$ to be irreducible.

Definition 2 Let $\mathcal{A}$ be an m-order and n-dimensional tensor. We define $\sigma(\mathcal{A})$ the Z-spectrum of $\mathcal{A}$ by the set of all Z-eigenvalues of $\mathcal{A}$. Assume $\sigma(\mathcal{A}) \neq \emptyset$, then the $\mathrm{Z}$-spectral radius of $\mathcal{A}$ is denoted by

$$
\rho(\mathcal{A})=\max \{|\lambda|: \lambda \in \sigma(\mathcal{A})\} .
$$

Let $N=\{1,2, \ldots, n\}$. In 2013, Chang et al. gave the following bound for the Z-eigenvalues of an $m$-order $n$-dimensional tensor $\mathcal{A}$.

Theorem 1 Let $\mathcal{A}$ be an m-order and n-dimensional tensor. Then

$$
\rho(\mathcal{A}) \leq \sqrt{n} \max _{i \in N} \sum_{i_{2}, \ldots, i_{m}=1}^{n}\left|a_{i i_{2} \ldots i_{m}}\right| .
$$

For the positively homogeneous operators, Song and Qi (2013) studied the relationship between the Gelfand formula and the spectral radius as well as the upper bound of the spectral radius. From Corollary 4.5 in Song and Qi (2013), we can get the following result:

Theorem 2 Let $\mathcal{A}$ be an m-order and n-dimensional tensor. Then

$$
\rho(\mathcal{A}) \leq \max _{i \in N} \sum_{i_{2}, \ldots, i_{m}=1}^{n}\left|a_{i i_{2} \ldots i_{m}}\right| .
$$

We shall denote the set of all $m$ th order $n$ dimensional tensors by $\mathbb{R}^{[m, n]}$, and the set of all nonnegative (or, respectively, positive) $m$ th order $n$ dimensional tensors by $\mathbb{R}_{+}^{[m, n]}$ (or, respectively, $\mathbb{R}_{++}^{[m, n]}$ ). If the tensor is positive, He and Huang gave the following Z-eigenpair bound (see Theorem 2.7 of He and Huang 2014):

Theorem 3 Suppose that $\mathcal{A}=\left(a_{i_{1} i_{2} \ldots i_{m}}\right) \in \mathbb{R}_{++}^{[m, n]}$ is an irreducible weakly symmetric tensor. Then

$$
\rho(\mathcal{A}) \leq R-l(1-\theta)
$$

where $R_{i}=\sum_{i_{2}, \ldots, i_{m}=1}^{n}\left|a_{i i_{2} \ldots i_{m}}\right|$

$$
R=\max _{i \in N} R_{i}, r=\min _{i \in N} R_{i}, \quad l=\min _{i_{1}, \ldots, i_{m}} a_{i_{1} \ldots i_{m}}, \quad \theta=\left\{\frac{r}{R}\right\}^{\frac{1}{m}} .
$$

Li et al. obtained the following upper bound (see Theorem 3.5 of Li et al. 2015):

Theorem 4 Suppose that $\mathcal{A}=\left(a_{i_{1} i_{2} \ldots i_{m}}\right) \in \mathbb{R}_{+}^{[m, n]}$ is an irreducible weakly symmetric tensor. Then 


$$
\rho(\mathcal{A}) \leq \max _{i, j}\left\{r_{i}+a_{i j \ldots j}\left(\delta^{-\frac{m-1}{m}}-1\right)\right\}
$$

where

$$
\delta=\frac{\min _{i, j} a_{i j \ldots j}}{r-\min _{i, j} a_{i j \ldots j}}\left(\gamma^{\frac{m-1}{m}}-\gamma^{\frac{1}{m}}\right)+\gamma, \quad \gamma=\frac{R-\min _{i, j} a_{i j \ldots j}}{r-\min _{i, j} a_{i j \ldots j}} .
$$

A real tensor of order $m$ dimension $n$ is called the unit tensor, if its entries are $\delta_{i_{1} \ldots i_{m}}$ for $i_{1}, \ldots, i_{m} \in N$, where

$$
\delta_{i_{1} \ldots i_{m}}= \begin{cases}1, & \text { if } i_{1}=\cdots=i_{m} \\ 0, & \text { otherwise. }\end{cases}
$$

And we define

$$
r_{i}(\mathcal{A})=\sum_{\delta_{i i_{2} \ldots i_{m}}=0}\left|a_{i i_{2} \ldots i_{m}}\right|, \quad r_{i}^{j}(\mathcal{A})=\sum_{\substack{\delta_{i i_{2} \ldots i_{m}}=0, \delta_{j i_{2} \ldots i_{m}}=0}}\left|a_{i i_{2} \ldots i_{m}}\right|=r_{i}(\mathcal{A})-\left|a_{i j \ldots j}\right| .
$$

He gave the following upper bound (see Theorem 3.3 of He 2016):

Theorem 5 Suppose that $\mathcal{A}=\left(a_{i_{1} i_{2} \ldots i_{m}}\right) \in \mathbb{R}_{+}^{[m, n]}$ is an irreducible weakly symmetric tensor. Then

$$
\rho(\mathcal{A}) \leq \max _{i, j \in N, j \neq i} \frac{1}{2}\left\{a_{i \ldots i}+a_{j \ldots j}+r_{i}^{j}(\mathcal{A})+\Theta_{i, j}^{\frac{1}{2}}(\mathcal{A})\right\},
$$

where

$$
\Theta_{i, j}(\mathcal{A})=\left(a_{i \ldots i}-a_{j \ldots j}+r_{i}^{j}(\mathcal{A})\right)^{2}+4 a_{i j \ldots j} r_{j}(\mathcal{A}) .
$$

Our goal in this paper is to show some tighter upper bounds for the largest Z-eigenvalue of a nonnegative tensor. In section "Main results", some new upper bounds for the largest Z-eigenvalue are obtained, which are tighter than the results in Theorems 1-5 (Chang et al. 2013; Song and Qi 2013; He and Huang 2014; Li et al. 2015; He 2016).

\section{Main results}

In this section, we consider some new upper bounds for the largest Z-eigenvalue of a nonnegative tensor.

A tensor $\mathcal{A}$ is called weakly symmetric if the associated homogeneous polynomial $\mathcal{A} x^{m}$ satisfies

$$
\nabla \mathcal{A} x^{m}=m \mathcal{A} x^{m-1} .
$$

This concept was first introduced and used by Chang et al. (2013) for studying the properties of $Z$-eigenvalue of nonnegative tensors and presented the following Perron-Frobenius Theorem for the $Z$-eigenvalue of nonnegative tensors. 
Lemma 1 Suppose that $\mathcal{A}=\left(a_{i_{1} i_{2} \ldots i_{m}}\right) \in \mathbb{R}_{+}^{[m, n]}$ is an irreducible weakly symmetric tensor, then the spectral radius $\rho(\mathcal{A})$ is a positive Z-eigenvalue with a positive Z-eigenvector.

Based on the lemma, we give our main results as follows.

Theorem 6 Suppose that $\mathcal{A}=\left(a_{i_{1} i_{2} \ldots i_{m}}\right) \in \mathbb{R}_{+}^{[m, n]}$ is an irreducible weakly symmetric tensor. Then

$$
\rho(\mathcal{A}) \leq \max _{i \in N} \min _{j \in N, j \neq i} \frac{1}{2}\left\{a_{i \ldots i}+a_{j \ldots j}+r_{i}^{j}(\mathcal{A})+\Theta_{i, j}^{\frac{1}{2}}(\mathcal{A})\right\},
$$

where

$$
\Theta_{i, j}(\mathcal{A})=\left(a_{i \ldots i}-a_{j \ldots j}+r_{i}^{j}(\mathcal{A})\right)^{2}+4 a_{i j \ldots j} r_{j}(\mathcal{A}) .
$$

Proof First, Let $x=\left(x_{1}, \ldots, x_{n}\right)^{T}$ be an Z-eigenvector of $\mathcal{A}$ corresponding to $\rho(\mathcal{A})$, that is,

$$
\mathcal{A} x^{m-1}=\rho(\mathcal{A}) x,
$$

Assume $0<x_{t}=\max _{i \in N} x_{i}$, then, for any $s \neq t$, by using $x_{t}^{m-1} \leq x_{t}, x_{s}^{m-1} \leq x_{s}$, we get

$$
\begin{gathered}
\left(\rho(\mathcal{A})-a_{t \ldots t}\right) x_{t}^{m-1}-a_{t s \ldots s} x_{s}^{m-1} \leq \sum_{\substack{\delta_{t i_{2} \ldots i_{m}}=0, \delta_{s i_{2} \ldots i_{m}}=0}} a_{t i_{2} \ldots i_{m}} x_{i_{2}} \ldots x_{i_{m}}, \\
\left(\rho(\mathcal{A})-a_{s . . . s}\right) x_{s}^{m-1}-a_{s t \ldots t} x_{t}^{m-1} \leq \sum_{\substack{\delta_{t i_{2} \ldots m_{m}}=0, \delta_{s i_{2} \ldots i_{m}}=0}} a_{s i_{2} \ldots i_{m}} x_{i_{2}} \ldots x_{i_{m}} .
\end{gathered}
$$

From Corollary 4.10 in Chang et al. (2013), we have

$$
\rho(\mathcal{A})-a_{i \ldots i} \geq 0, \quad i=1, \ldots, n .
$$

Then, from (7) and (8), we obtain, we obtain

$$
\begin{aligned}
\left(\left(\rho(\mathcal{A})-a_{s \ldots .}\right)\left(\rho(\mathcal{A})-a_{t \ldots t}\right)-a_{s t \ldots t} a_{t s \ldots s}\right) x_{t}^{m-1} \leq\left(\rho(\mathcal{A})-a_{s . \ldots s}\right) \sum_{\substack{\delta_{t i_{2} \ldots i_{m}}=0, \delta_{s i_{2} \ldots i_{m}}=0}} a_{t i_{2} \ldots i_{m}} x_{i_{2}} \ldots x_{i_{m}} \\
+a_{t s \ldots s} \sum_{\substack{\delta_{t t_{2} \ldots i_{m}}=0, \delta_{s 2_{2} \ldots i_{m}}=0}} a_{s i_{2} \ldots i_{m}} x_{i_{2}} \ldots x_{i_{m}} .
\end{aligned}
$$

Recalling that $0<x_{t}=\max _{i \in N} x_{i}$, we have

$$
\begin{aligned}
& \left(\rho(\mathcal{A})-a_{s \ldots .}\right)\left(\rho(\mathcal{A})-a_{t \ldots t}\right)-a_{s t \ldots t} a_{t s \ldots s} \leq\left(\rho(\mathcal{A})-a_{s . . . s}\right) \sum_{\substack{\delta_{t i_{2} \ldots m_{m}}=0, \delta_{s i_{2} \ldots i_{m}}=0}} a_{t i_{2} \ldots i_{m}} \frac{x_{i_{2}}}{x_{t}} \ldots \frac{x_{i_{m}}}{x_{t}} \\
& +a_{t s \ldots s} \sum_{\substack{\delta_{t i_{2} \ldots i_{m}}=0, \delta_{s i_{2} \ldots i_{m}}=0}} a_{s i_{2} \ldots i_{m}} \frac{x_{i_{2}}}{x_{t}} \ldots \frac{x_{i_{m}}}{x_{t}} \\
& \leq\left(\rho(\mathcal{A})-a_{s . . . s}\right) r_{t}^{s}(\mathcal{A})+a_{t s . . .} r_{s}^{t}(\mathcal{A}) .
\end{aligned}
$$


Therefore

$$
\rho(\mathcal{A}) \leq \frac{1}{2}\left\{a_{t \ldots t}+a_{s \ldots s}+r_{t}^{s}(\mathcal{A})+\Theta_{t, s}^{\frac{1}{2}}(\mathcal{A})\right\} .
$$

This must be true for every $s \neq t$, then, we get

$$
\rho(\mathcal{A}) \leq \min _{j \in N, j \neq t} \frac{1}{2}\left\{a_{t \ldots t}+a_{j \ldots j}+r_{t}^{j}(\mathcal{A})+\Theta_{t, j}^{\frac{1}{2}}(\mathcal{A})\right\} .
$$

And this could be true for any $t \in N$, that is

$$
\rho(\mathcal{A}) \leq \max _{i \in N} \min _{j \in N, j \neq i} \frac{1}{2}\left\{a_{i \ldots i}+a_{j \ldots j}+r_{i}^{j}(\mathcal{A})+\Theta_{i, j}^{\frac{1}{2}}(\mathcal{A})\right\} .
$$

Thus, we complete the proof.

Remark 1 Obviously, we can get

$$
\max _{i \in N} \min _{j \in N, j \neq i} \frac{1}{2}\left\{a_{i \ldots i}+a_{j \ldots j}+r_{i}^{j}(\mathcal{A})+\Theta_{i, j}^{\frac{1}{2}}(\mathcal{A})\right\} \leq \max _{i, j \in N, j \neq i} \frac{1}{2}\left\{a_{i \ldots i}+a_{j \ldots . j}+r_{i}^{j}(\mathcal{A})+\Theta_{i, j}^{\frac{1}{2}}(\mathcal{A})\right\} .
$$

That is to say, the bound in Theorem 6 is always better than the result in Theorem 5 .

We denote

$$
\begin{aligned}
& \Delta_{i}=\left\{\left(i_{2}, i_{3}, \ldots, i_{m}\right): i_{j}=i \quad \text { for some } j \in\{2, \ldots, m\}\right\}, \quad \text { where } i, i_{2}, \ldots, i_{m} \in N \\
& \bar{\Delta}_{i}=\left\{\left(i_{2}, i_{3}, \ldots, i_{m}\right): i_{j} \neq i \text { for any } j \in\{2, \ldots, m\}\right\}, \quad \text { where } i, i_{2}, \ldots, i_{m} \in N .
\end{aligned}
$$

And let

$$
r_{i}^{\Delta_{j}}(\mathcal{A})=\sum_{\substack{\left(i_{2}, \ldots, i_{m}\right) \in \Delta_{j} \\ \delta_{i i_{2} \ldots i_{m}}=0}}\left|a_{i i_{2} \ldots i_{m}}\right|, \quad r_{i}^{\bar{\Delta}_{j}}(\mathcal{A})=\sum_{\left(i_{2}, \ldots, i_{m}\right) \in \bar{\Delta}_{j}}\left|a_{i i_{2} \ldots i_{m}}\right| .
$$

Then, $r_{i}(\mathcal{A})=r_{i}^{\Delta_{j}}+r_{i}^{\bar{\Delta}_{j}}(\mathcal{A})$.

Theorem 7 Suppose that $\mathcal{A}=\left(a_{i_{1} i_{2} \ldots i_{m}}\right) \in \mathbb{R}_{+}^{[m, n]}$ is an irreducible weakly symmetric tensor. Then

$$
\rho(\mathcal{A}) \leq \max _{i \in N} \min _{j \in N, j \neq i} \frac{1}{2}\left\{a_{i \ldots i}+a_{j \ldots j}+r_{i}^{\bar{\Delta}_{j}}(\mathcal{A})+\Omega_{i, j}^{\frac{1}{2}}(\mathcal{A})\right\}
$$

where

$$
\Omega_{i, j}(\mathcal{A})=\left(a_{i \ldots i}-a_{j \ldots j}+r_{i}^{\bar{\Delta}_{j}}(\mathcal{A})\right)^{2}+4 r_{i}^{\Delta_{j}}(\mathcal{A}) r_{j}(\mathcal{A})
$$

Proof First, Let $x=\left(x_{1}, \ldots, x_{n}\right)^{T}$ be an Z-eigenvector of $\mathcal{A}$ corresponding to $\rho(\mathcal{A})$, that is, 


$$
\mathcal{A} x^{m-1}=\rho(\mathcal{A}) x
$$

Assume $0<x_{t}=\max _{i \in N} x_{i}$, then, we can get

$$
\begin{aligned}
\rho(\mathcal{A}) x_{t} & =\sum_{\left(i_{2}, \ldots, i_{m}\right) \in \Delta_{s}} a_{t i_{2} \ldots i_{m}} x_{i_{2}} \ldots x_{i_{m}}+\sum_{\left(i_{2}, \ldots, i_{m}\right) \in \bar{\Delta}_{s}} a_{t i_{2} \ldots i_{m}} x_{i_{2}} \ldots x_{i_{m}} \\
& \leq \sum_{\left(i_{2}, \ldots, i_{m}\right) \in \Delta_{s}} a_{t i_{2} \ldots i_{m}} x_{s}+\sum_{\left(i_{2}, \ldots, i_{m}\right) \in \bar{\Delta}_{s}} a_{t i_{2} \ldots i_{m}} x_{i_{2}} \ldots x_{i_{m}} .
\end{aligned}
$$

That is

$$
\left(\rho(\mathcal{A})-a_{t \ldots t}\right) x_{t}-r_{t}^{\Delta_{s}}(\mathcal{A}) x_{s} \leq \sum_{\substack{\left(i_{2}, \ldots, i_{m}\right) \in \bar{\Delta}_{s} \\ \delta_{t i_{2} \ldots i_{m}}=0}} a_{t i_{2} \ldots i_{m}} x_{i_{2}} \ldots x_{i_{m}}
$$

Similarly, we can get

$$
\left(\rho(\mathcal{A})-a_{s \ldots s}\right) x_{s}-r_{s}^{\Delta_{t}}(\mathcal{A}) x_{t} \leq \sum_{\left(i_{2}, \ldots, i_{m}\right) \in \bar{\Delta}_{t}} a_{s i_{2} \ldots i_{m}} x_{i_{2}} \ldots x_{i_{m}} .
$$

From Corollary 4.10 in Chang et al. (2013), we have

$$
\rho(\mathcal{A})-a_{i \ldots i} \geq 0, \quad i=1, \ldots, n \text {. }
$$

Then, from (13) and (14), we obtain, we obtain

$$
\begin{aligned}
& \left(\left(\rho(\mathcal{A})-a_{s \ldots s}\right)\left(\rho(\mathcal{A})-a_{t \ldots t}\right)-r_{t}^{\Delta_{s}}(\mathcal{A}) r_{s}^{\Delta_{t}}(\mathcal{A})\right) x_{t} \leq\left(\rho(\mathcal{A})-a_{s \ldots s}\right) \sum_{\substack{\left(i_{2}, \ldots, i_{m}\right) \in \bar{\Delta}_{s} \\
\delta_{t i_{2} \ldots i_{m}}=0}} a_{t i_{2} \ldots i_{m}} x_{i_{2}} \ldots x_{i_{m}} \\
& +r_{t}^{\Delta_{s}}(\mathcal{A}) \sum_{\left(i_{2}, \ldots, i_{m}\right) \in \bar{\Delta}_{t}} a_{s i_{2} \ldots i_{m}} x_{i_{2}} \ldots x_{i_{m}} .
\end{aligned}
$$

Recalling that $0<x_{t}=\max _{i \in N} x_{i}$, we have

$$
\left(\rho(\mathcal{A})-a_{s \ldots s}\right)\left(\rho(\mathcal{A})-a_{t \ldots t}\right)-r_{t}^{\Delta_{s}}(\mathcal{A}) r_{s}^{\Delta_{t}}(\mathcal{A}) \leq\left(\rho(\mathcal{A})-a_{s \ldots s}\right) r_{t}^{\bar{\Delta}_{s}}(\mathcal{A})+r_{t}^{\Delta_{s}}(\mathcal{A}) r_{s}^{\bar{\Delta}_{t}}(\mathcal{A}) .
$$

Therefore

$$
\rho(\mathcal{A}) \leq \frac{1}{2}\left\{a_{t \ldots t}+a_{s \ldots s}+r_{t}^{\bar{\Delta}_{s}}(\mathcal{A})+\Omega_{t, s}^{\frac{1}{2}}(\mathcal{A})\right\} .
$$

This must be true for every $s \neq t$, then, we get

$$
\rho(\mathcal{A}) \leq \min _{j \in N, j \neq t} \frac{1}{2}\left\{a_{t \ldots t}+a_{j \ldots j}+r_{t}^{\bar{\Delta}_{s}}(\mathcal{A})+\Omega_{t, j}^{\frac{1}{2}}(\mathcal{A})\right\} .
$$

And this could be true for any $t \in N$, that is

$$
\rho(\mathcal{A}) \leq \max _{i \in N} \min _{j \in N, j \neq i} \frac{1}{2}\left\{a_{i \ldots i}+a_{j \ldots j}+r_{i}^{\bar{\Delta}_{j}}(\mathcal{A})+\Omega_{i, j}^{\frac{1}{2}}(\mathcal{A})\right\} .
$$

Thus, we complete the proof. 
Remark 2 Let $\Pi_{i}$ be a nonempty proper subset of $\Delta_{i}$, we have that for $\left(i_{2}, \ldots, i_{m}\right) \in \Pi_{i}$,

$$
r_{i}^{\Delta_{i}}(\mathcal{A})=r_{i}^{\Pi_{i}}(\mathcal{A})+r_{i}^{\bar{\Pi}_{i}}(\mathcal{A})
$$

Similar to the proof of Theorem 7, we can get

$$
\rho(\mathcal{A}) \leq \min _{\Pi_{i} \in \Delta_{i}} \max _{i \in N} \min _{j \in N, j \neq i} \frac{1}{2}\left\{a_{i \ldots i}+a_{j \ldots j}+r_{i}^{\bar{\Pi}_{j}}(\mathcal{A})+\Upsilon_{i, j}^{\frac{1}{2}}(\mathcal{A})\right\}
$$

where

$$
\Upsilon_{i, j}(\mathcal{A})=\left(a_{i \ldots i}-a_{j \ldots j}+r_{i}^{\bar{\Pi}_{j}}(\mathcal{A})\right)^{2}+4 r_{i}^{\Pi_{j}}(\mathcal{A}) r_{j}(\mathcal{A})
$$

which is always better than the result in Theorem 6 .

Example 1 We now show the efficiency of the new upper bounds in Theorems 6 and 7 by the following example. Consider the tensor $\mathcal{A}=\left(a_{i j k}\right)$ and of order 3 dimension 3 with entries defined as follows:

$$
a_{111}=\frac{1}{2}, \quad a_{222}=1, \quad a_{333}=3, \quad \text { and } \quad a_{i j k}=\frac{1}{3} \quad \text { elsewhere }
$$

By Theorem 1, we have

$$
\rho(\mathcal{A}) \leq 9.8150 .
$$

By Theorem 2, we have

$$
\rho(\mathcal{A}) \leq 5.6667 .
$$

By Theorem 3, we have

$$
\rho(\mathcal{A}) \leq 5.6079 .
$$

By Theorem 4, we have

$$
\rho(\mathcal{A}) \leq 5.5494 .
$$

By Theorem 5, we have

$$
\rho(\mathcal{A}) \leq 5.5296 .
$$

By Theorem 6, we have

$$
\rho(\mathcal{A}) \leq 5.5107 .
$$

By Theorem 7, we have

$$
\rho(\mathcal{A}) \leq 5.3654 .
$$

This example shows that the bound in Theorem 7 is the best among the known bounds. 


\section{Conclusion}

In this paper, we presented some bounds for the largest Z-eigenvalue of an irreducible weakly symmetric and nonnegative tensor. These bounds are always sharper than the bounds in Chang et al. (2013), Song and Qi (2013), He and Huang (2014), Li et al. (2015), He (2016).

\section{Authors' contributions}

All authors contributed equally to this work. All authors read and approved the final manuscript.

\section{Acknowledgements}

This work was supported by the Doctoral Scientific Research Foundation of Zunyi Normal College (No. BS[2015]09); Science and technology Foundation of Guizhou province (Qian ke he Ji Chu [2016]1161). Liu is supported by National Natural Science Foundations of China (Grants nos. 71461027); Science and technology talent training object of Guizhou province outstanding youth (Qian ke he ren zi [2015]06); Guizhou province natural science foundation in China (Qian Jiao He KY [2014]295); 2013, 2014 and 2015 Zunyi 15851 talents elite project funding; Zhunyi innovative talent team(Zunyi $\mathrm{KH}(2015) 38$ ). Tian is supported by the Science and Technology fund Project of GZ (Qian ke he J Zi [2015]2147, Qian jiao he KY[2015]451). Ke is supported by the Science and Technology fund Project of GZ (Qian Ke He J Zi LKZS [2012]08). Li is supported by the Science and Technology fund Project of GZ (Qian Ke He LH[2015]7047).

\section{Competing interests}

The authors declare that they have no competing interests.

Received: 7 June 2016 Accepted: 21 September 2016

Published online: 06 October 2016

\section{References}

Chang KC, Pearson K, Zhang T (2009) On eigenvalue problems of real symmetric tensors. J Math Anal Appl 350:416-422 Chang KC, Pearson K, Zhang T (2013) Some variational principles for Z-eigenvalues of nonnegative tensors. Linear Algebra Appl 438:4166-4182

Chang KC, Qi L, Zhou G (2010) Singular values of a real rectangular tensor. J Math Anal Appl 37:284-294

Chang KC, Zhang T, Pearson K (2008) Perron-Frobenius theorem for nonnegative tensors. Commun Math Sci 6:507-520

He J (2016) Bounds for the largest eigenvalue of nonnegative tensors. J Comput Anal Appl 20:1290-1301

He J, Huang TZ (2014) Upper bound for the largest Z-eigenvalue of positive tensors. Appl Math Lett 38:110-114

Hu S, Huang Z (2012) Theorems of the alternative for inequality systems of real polynomials. J. Optim. Theory Appl 154:1-16

Hu S, Huang Z, Qi L (2013) Finding the extreme z-eigenvalues of tensors via a sequential semidefinite programming method. Numer Linear Algebra Appl 20:972-984

Li C, Chen Z, LiY (2015) A new eigenvalue inclusion set for tensors and its applications. Linear Algebra Appl 481:36-53

Li C, Li Y, Kong X (2014) New eigenvalue inclusion sets for tensors. Numer Linear Algebra Appl 21:39-50

Li W, Liu D, Vong S (2015) Z-eigenpair bounds for an irreducible nonnegative tensor. J Comput Anal Appl 483:182-199

Lim LH (2005) Singular values and eigenvalues of tensors: A variational approach. In: Proceedings of the IEEE international workshop on computational advances in multi-sensor adaptive processing, pp 129-132. CAMSAP 05

Ng MK, Qi L, Zhou G (2009) Finding the largest eigenvalue of a non-negative tensor. SIAM J Matrix Anal Appl 31:1090-1099

Qi L (2005) Eigenvalues of a real supersymmetric tensor. J Symb Comput 40:1302-1324

Qi L (2007) Eigenvalues and invariants of tensor. J Math Anal Appl 325:1363-1377

Song Y, Qi L (2013) Spectral properties of positively homogeneous operators induced by higher order tensors. SIAM J Matrix Anal Appl 34:1581-1595

Yang Y, Yang Q (2010) Further results for Perron-Frobenius theorem for nonnegative tensors. SIAM J Matrix Anal Appl 31:2517-2530

Yang Y, Yang Q (2011) Further results for Perron-Frobenius theorem for nonnegative tensors II. SIAM J Matrix Anal Appl 32:1236-1250

Zhou G, Caccetta L, Qi L (2013) Convergence of an algorithm for the largest singular value of a nonnegative rectangular tensor. Linear Algebra Appl 438:959-968 